\section{New CPD requirements announced}

The General Dental Council (GDC) has agreed changes to continuing professional development (CPD) which will come into force in 2018.

The changes include the requirement for each member of the dental team to have a personal development plan (PDP); changes to the number of hours of verifiable CPD dentists and dental care professionals (DCPs) have to complete; and the end of the requirement to keep records of general (non-verifiable) CPD.

In the current $\mathrm{CPD}$ scheme, dentists are required to complete 250 hours of CPD over five years, of which 75 are verifiable. In the new scheme, known as Enhanced CPD or ECPD, they will be required to complete 100 hours of verifiable CPD in the five-year period.

In the current CPD scheme, DCPs are required to complete 150 hours of CPD over five years, of which 50 are verifiable. In the new scheme, dental hygienists, dental therapists, clinical dental technicians and orthodontic therapists will be required to complete 75 hours of verifiable $\mathrm{CPD}$ over the five-year period, and dental nurses and dental technicians are required to complete 50 hours.

Dental professionals will also have to make an annual declaration of the amount of CPD activity they have completed.

The changes will happen in January 2018 for dentists and in August 2018 for DCPs.

The BDJ will continue to offer BDA members 48 hours of verifiable $C P D$ a year. DCPs can complete ten free hours of verifiable CPD a year through reading $\mathrm{BDJ}$ Team.

\section{Down on the beach with your dentist}

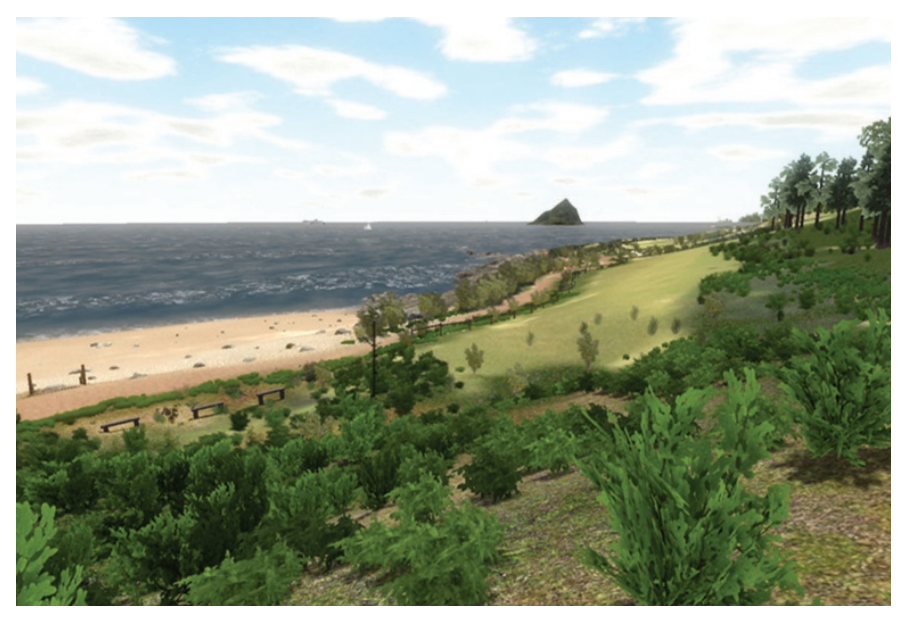

Researchers from the Universities of Plymouth, Exeter and Birmingham have found that a virtual reality walk on a beach improves patient experiences of common dental procedures such as fillings and extractions. The virtual beach was shown to have better outcomes than a virtual tour of an urban environment, or no virtual input at all.

In a study published last month in the journal Environment and Behavior, a team of researchers at the Universities of Plymouth, Exeter and Birmingham worked with Torrington Dental Practice in Devon to find out whether virtual experiences could improve the dental patient's experience during routine dental procedures, such as fillings and tooth extractions.

What the patient sees can be viewed here: https://youtu.be/ n5kjETt8cZI.

Dr Karin Tanja-Dijkstra, the lead author of the study, said: 'The use of virtual reality in healthcare settings is on the rise but we need more rigorous evidence of whether it actually improves patient experiences. Our research demonstrates that under the right conditions, this technology can be used to help both patients and practitioners'.

The authors of the research stress that the type of virtual reality environment the patient visits is important. The beach experience, Virtual Wembury, was created by Professor Bob Stone and colleagues at the University of Birmingham, and the fact that only patients who visited Wembury, and not the virtual city, had better experiences than standard care is consistent with a growing body of work that shows that natural environments, and marine environments in particular, can help reduce stress and anxiety.

The Torrington Practice dentist involved in the research, Melissa Auvray, agreed: 'The level of positive feedback we got from patients visiting Virtual Wembury was fantastic. Of course, as dentists we do our very best to make the patient feel as comfortable as possible but we are always on the lookout for new ways to improve their experiences'.

1. Tanja-Dijkstra K, Pahl S, White M P et al. The soothing sea: a virtual coastal walk can reduce experienced and recollected pain. EAB 2017; 1-27. DOI: https://doi. org/10.1177/0013916517710077. 